\title{
Un sistema experto basado en minería de datos y programación entera lineal para soporte en la asignación de materias y diseño de horarios en educación superior
}

\section{(An expert system based on data mining and linear integer programming to support the timetabling design and courses assignment in higher education)}

\author{
Daniel Calle-López ${ }^{1}$, Javier Cornejo-Reyes ${ }^{1}$, Fernando Pesántez-Avilés ${ }^{1}$, Mónica Rodas- \\ Tobar $^{2}$, César Vásquez-Vásquez ${ }^{1}$, Vladimir Robles-Bykbaev ${ }^{1}$
}

\begin{abstract}
Resumen:
En general, varias de las organizaciones tienden a manejar la asignación de cargas horarias de sus empleados de manera tradicional (imposición de una jornada de trabajo de 8). Todo esto a fin de mantener cierto control sobre variables como asistencia y ausentismo del personal e incluso en algunos casos considerando aquello como sinónimo de eficacia laboral. Sin embargo, la nueva tendencia organizacional ha roto ciertos paradigmas con orientación mecanicista, pretendiendo crear un nuevo horizonte hacia la construcción de organizaciones orgánicas y dinámicas. Por lo expuesto, en este artículo se describe una propuesta de sistema experto basado en programación entera lineal y minería de datos para abordar el problema de asignación de materias y el diseño de horarios (considerado un problema NP-completo). Los resultados preliminares son alentadores, ya que han permitido realizar la asignación de materias en una base de 133.000 registros de docentes y generar horarios con un mínimo de coste computacional.
\end{abstract}

Palabras clave: programación entera lineal; minería de datos; diseño de horarios; asignación de materias; educación superior.

\begin{abstract}
:
Commonly, the most of organizations tend to manage the timetables of their employees according to traditional guidelines (imposing an 8-hour work day). The primary objective of this approach is controlling some variables such as the attendance and absenteeism of the employees, and even, in some cases considering this situation as work efficiency. However, the new organizational tendencies have broken specific paradigms based on mechanistic orientation, trying to create a new horizon towards the construction of organic and dynamic organizations. For these reasons, in this paper, we present an expert system based on integer linear programming and data mining with the aim of addressing the problem of assigning courses to teachers and timetabling (considered an NP-complete problem). The preliminary results are encouraging, given that the system was able to assign courses on a database consisting off 133.000 registers of teachers, and at the same time, generate timetables with minimal computational costs.
\end{abstract}

Keywords: integer linear programming; data mining; timetabling design; courses assignment; higher education.

\footnotetext{
${ }^{1}$ GI-IATa, Cátedra UNESCO Tecnologías de apoyo para la Incl. Educativa, Universidad Politécnica Salesiana, Cuenca - Ecuador (\{dcallel,jcornejor, fpesantez, cvasquez, vrobles\}@ups.edu.ec).

${ }^{2}$ Escuela de Psicología Organizacional, Universidad del Azuay, Cuenca - Ecuador (mrodast@uazuay.edu.ec).
} 


\section{Introducción}

Las universidades en particular, al ser su esencia la generación de conocimiento, se encuentran empeñadas en desarrollar estrategias en el ámbito de la gestión de lo académico, para asegurar, primero, la satisfacción laboral de sus cuerpos docentes y luego los resultados y logros académicos de sus alumnados. Se enfrentan al reto de armonizar la gestión horaria y lectiva además de las responsabilidades complementarias según el tipo de dedicación docente (CES, 2010); las características biográficas (edad, género, estado civil, maternidad, paternidad, lugar de vivienda) son también variables necesarias a la hora de definir los horarios de la docencia; por citar, en el caso de los profesores investigadores no es necesario asignar un horario establecido, al contrario, estos profesionales deben trabajar por resultados y avances de sus programas 0 proyectos de investigación.

Entre los aspectos más apreciativos por parte del profesorado universitario cuando les corresponde autovalorar su satisfacción tiene que ver con los horarios y la carga lectiva a ellos asignada para el ejercicio de sus tareas docentes. Considerando que las instituciones educativas como organización no manejan iguales lógicas que las empresas, sus directrices no pueden ser rígidas en cuanto al tema de gestión horaria, esto en función de diversas restricciones que se acumulan alrededor del propio ejercicio docente en relación incluso con factores biosociales del educador y del educando. La flexibilidad requerida en la gestión horaria docente ha de considerar que es común, en la actualidad, manejar públicos heterogéneos donde la presencia de estudiantes adultos y la de estudiantes jóvenes a tiempo parcial es habitual, la irrupción de sistemas y modalidades como la distancia, de convergencia de medios o en línea (online) ya no es novedad, al igual que la presencia de otros agentes formativos como profesionales y empresarios a los que se les favorece con gestiones horarios menos exigentes (Zabalza y Berraza, 2003).

La gestión horaria no solamente ha de verse desde la lógica de beneficio del enseñante, debe entenderse que esta afecta directamente al tema de rendimiento académico del estudiante, y es considerado un factor de orden institucional, al cual se le puede controlar, modificar en pro de los rendimientos (Garbanzo Vargas, 2007). Además, según el tipo de contrato y dedicación docente, sea este de medio tiempo, parcial, completo o de dedicación exclusiva, las tareas docentes interactúan a nivel de funciones universitarias, para unos la tarea es eminentemente formativa, mientras que para otros se mezcla con funciones de investigación, gestión y vinculación con la sociedad.

El sistema de recomendación horaria para el trabajo docente, al igual que otros sistemas inteligentes para la gestión del conocimiento (Sánchez, Comas y García, 2016), además de ubicar lo mejor posible a un docente en un horario específico de clase según sus variables biográficas y de orden institucional, permite la identificación de espacios de tiempo para otras acciones como la conformación de claustros docentes que interactúan alrededor de procesos de innovación educativa. En el Ecuador el Consejo de Educación Superior citando a Larrea (2016) y refiriéndose a las condiciones y factores de pertinencia de calidad en la educación superior, destaca el rol estratégico del talento humano para alcanzar la pertinencia en el contexto de calidad, e indica que los docentes han de "asumir sus nuevas funciones como profesores-investigadores, tutores del proceso de aprendizaje, creadores de ambientes de aprendizaje, fortalecimiento de sus ambientes de aprendizaje" (Larrera, 2016); por lo que la gestión efectiva de horarios del personal docente debe no solo ser igual de efectiva a nivel individual sino grupalmente y colectivamente acertada.

El problema de asignación de horarios en las universidades, como se ha mencionado, es de características multivalente y a consideración de Caballero y Arboleda (2012), radica en: 
"programar en un horizonte de planificación (generalmente una semana) las asignaturas que se dictan en un período académico determinado (año, trimestre o semestre), para las distintas asignaturas que las requieren, considerando los profesores necesarios en cada asignatura, los grupos de alumnos que toman un conjunto de asignaturas, los días o períodos disponibles, los salones requeridos de tal manera que pueda optimizar un conjunto de restricciones relacionadas con la organización del sistema de educación" (Caballero y Arboleda, 2012).

Por lo expuesto, en este artículo se presenta una propuesta para el desarrollo de un sistema experto que se fundamenta en minería de datos y programación entera lineal para realizar dos complejas tareas de la gestión universitaria: la asignación de cursos (materias o asignaturas) a docentes (considerando la pertinencia y perfil de los docentes) y la generación automática de horarios. Finalmente, es importante destacar que esta investigación constituye una versión extendida y ampliada del trabajo presentado en el Conferencia Internacional de Sistemas de Información y Ciencias de la Computación INCISCOS (International Conference on Information Systems and Computer Science) (Pesántez et al., 2017).

\section{Metodología}

El sistema presenta un modelo organizado en tres capas y módulos que responden a la necesidad de asignación inteligente de distributivos y horarios. Esta estructura nos permite realizar cambios en los módulos sin afectar al funcionamiento de las demás capas. A continuación se describe la funcionalidad de dichas capas (Figura 1):

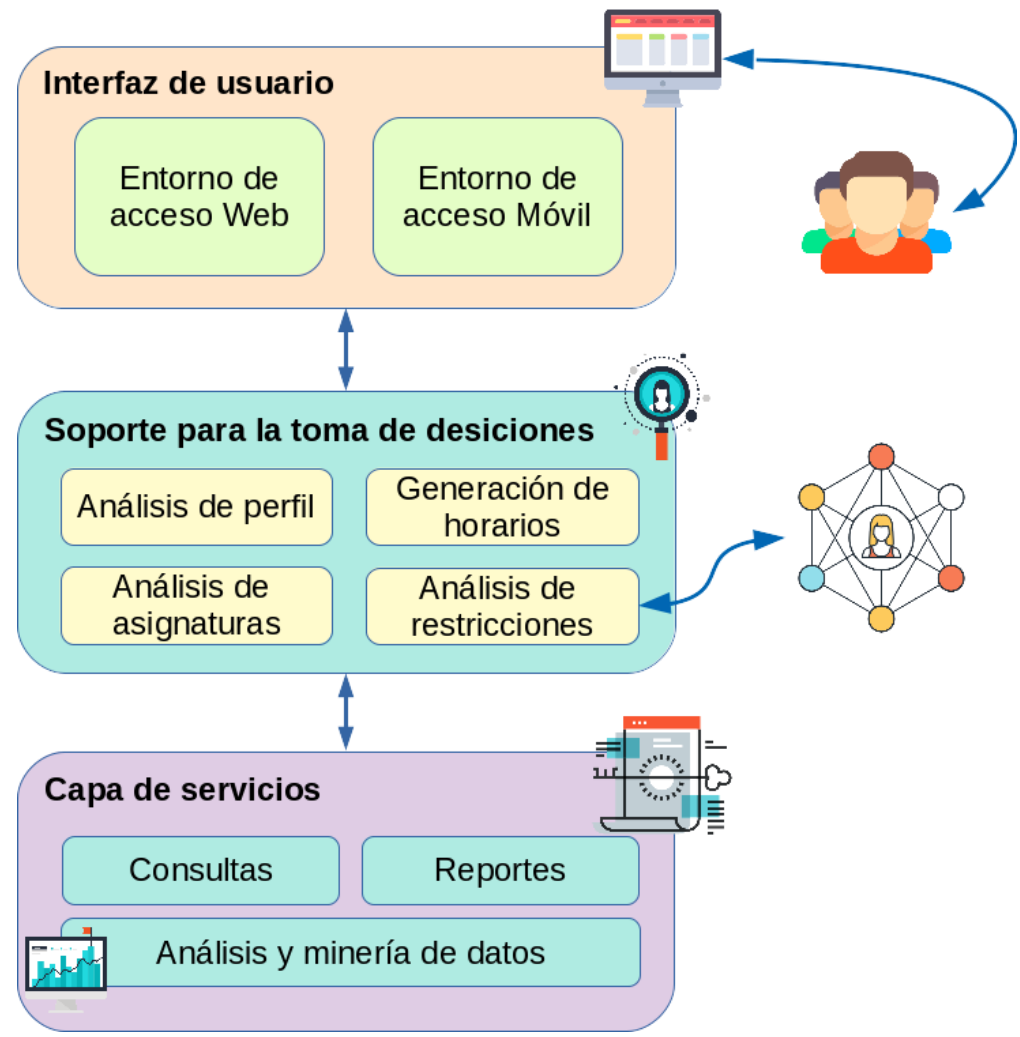

Figura 1. Arquitectura general del sistema propuesto para la generación automática de horarios y la asignación de cursos a docentes 


\subsection{Principales capas del sistema}

A continuación se describirán las capas del sistema con su respectiva funcionalidad.

Interfaz de usuario: contiene módulos que permiten la interacción de las autoridades encargadas de la asignación de horarios y distributivos con las diferentes funcionalidades del sistema. Permite la interacción y control efectivo del recomendador al tiempo que este retroalimenta al usuario con la información procesada en la capa de soporte a la toma de decisiones.

La aplicación móvil pretende brindar consultas rápidas, como alternativa a la interfaz web. Su naturaleza de aplicación móvil permite la implementación de funcionalidades extras, tales como notificaciones, sincronización e integración con aplicaciones nativas del dispositivo. Asimismo, la interfaz web presenta las recomendaciones de manera clara y entendible, con varias opciones de búsqueda avanzada.

Soporte para la toma de decisiones: los módulos de esta capa se encargan de procesar la información que proviene de la capa de servicios.

- Análisis de perfiles: este módulo asocia la clasificación de asignaturas y de docentes según lo especifica UNESCO en la Clasificación internacional normalizada de la educación (UNESCO, 2010). Aquí se comparan las clasificaciones tanto del perfil profesional y personal del docente con los de la asignatura definida dentro del currículo de la institución educativa.

- Análisis de asignaturas: este componente se conforma de dos subprocesos, el análisis de rendimiento docente para asignaturas y el subproceso de análisis de históricos docente-asignatura que evalúan el desempeño que ha tenido un docente $D$ con una asignatura $A$ durante toda su experiencia como docente.

- Generación de horarios: este componente está desarrollado con Algebra Lineal y Programación Entera Lineal para la resolución efectiva y generación de horarios de clases. Se toman las restricciones y el sistema penaliza a aquellos horarios que al momento de ser evaluados mediante la función objetivo no cumplen con los requerimientos con valores extremadamente negativos, de modo que al momento de minimizar, el modelo por sí solo se encargará de filtrar aquellos horarios que se han sabido adaptar de mejor manera a las restricciones impuestas.

- Análisis de restricciones: en este módulo se aplican validaciones que permiten verificar la estructura y sintaxis de las restricciones introducidas por los directores de carrera, de tal manera que puedan ser correctamente interpretadas por el módulo de generación de horarios.

Capa de servicios: abstrae la implementación interna referente a las consultas en los repositorios de datos institucionales para los módulos de soporte a la toma de decisiones y centraliza el acceso a datos y funciones. Esto permite que las capas superiores sean agnósticas a la estructura interna de la institución y esta pueda cambiar libremente de versiones siempre y cuando los servicios mantengan la misma estructura.

Esquema de implementación del sistema: el enfoque descrito en la Figura 2 ayuda a los desarrolladores en varios aspectos de desarrollo, la integración y entrega continua permiten al desarrollador ejecutar pruebas automatizadas sobre las diferentes versiones, esto ayuda a evitar problemas típicos de integración al combinar los cambios sobre la rama principal. 


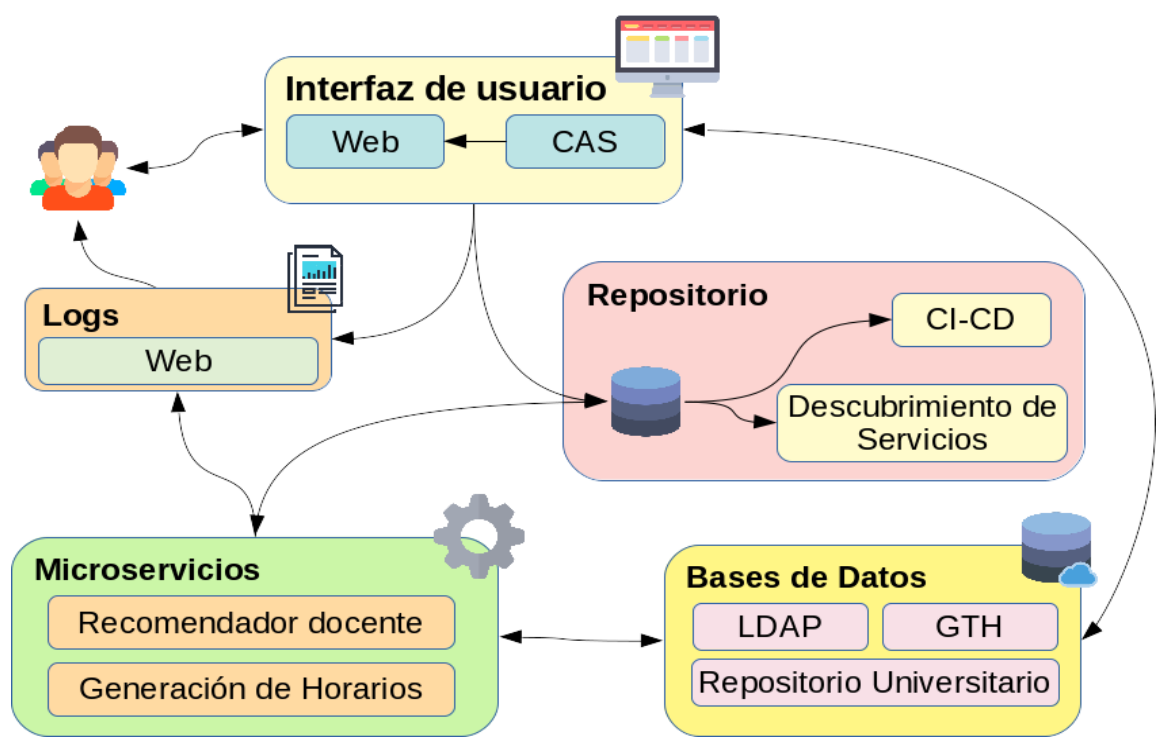

Figura 2. Esquema de implementación de la propuesta desarrollada

La ventaja de tener pruebas automatizadas para todas las versiones que se compilen del sistema nos da la certeza que los cambios que se hayan realizado se puedan efectuar rápida y de manera sostenible. En otras palabras, además de tener una automatización del proceso de compilación y pruebas se puede liberar el sistema de manera automática e implementar cualquier versión con un esfuerzo mínimo.

\subsection{Determinación de pares docente-asignatura:}

Los pares docente-asignatura representan una relación de pertinencia en base a la suma ponderada de tres componentes: histórico docente, evaluación estudiantil y relación de perfil docente-asignatura. El valor de pertinencia se calcula mediante la ecuación (1):

$$
\begin{gathered}
R(\vec{D}, \vec{A})=\frac{p_{1}}{\max \left(\phi_{k}\right)} \cdot \frac{\sum_{\forall k} \frac{\phi_{k}(D, A)}{1+\exp (k-N)}}{\sum_{\forall k} \frac{1}{1+\exp (k-N)}}+p_{2} \cdot \frac{|\vec{D}| \vee|\vec{A}|}{\max (D \vee A)}+p_{3} \cdot P(\vec{T}, \vec{A}) \\
P\left(\vec{T}_{D}, \vec{A}\right)=\left\{\begin{array}{cc}
1 & \overrightarrow{T_{D}} \in \operatorname{Dominio}(\vec{A}) \\
0.75 & \overrightarrow{T_{D}} \in \operatorname{SubArea}(\vec{A}) \\
0.25 & \overrightarrow{T_{D}} \in \operatorname{Area}(\vec{A}) \\
0 & -
\end{array}\right.
\end{gathered}
$$

Dónde:

- $\max \left(\phi_{k}\right)$ es la nota máxima de la evaluación docente

- $\quad k$ representa el número de período lectivo a evaluar

- $\quad \phi_{k}(D, A)$ resultado de la evaluación de un docente $D$ con una materia $A$ para un período lectivo $k$

- $\quad N$ constante que indica el número de períodos hacia atrás que serán tomados en cuenta para el cálculo de pertinencia

- $|\vec{D}| \vee|\vec{A}|$ representa la cantidad de docentes o asignaturas seleccionados para la consulta actual 
- $\quad \max (D \vee A)$ es un factor de normalización que se aplica cuando se recomienda los docentes que pueden dictar cierta asignatura o viceversa

- $\quad \overrightarrow{T_{D}}$ es un vector de títulos de tercer nivel o superiores correspondientes a un docente $D$

- $\quad \vec{T}_{D} \in \operatorname{Dominio}(\vec{A})$ indica que la clasificación de los títulos del docente coinciden con el dominio de las asignaturas, de acuerdo con la clasificación CINE (UNESCO, 2010)

- $\quad \vec{T}_{D} \in \operatorname{SubArea}(\vec{A})$ indica que la clasificación de los títulos del docente coinciden con el subárea de las asignaturas, de acuerdo con la clasificación CINE (UNESCO, 2010)

- $\quad \vec{T}_{D} \in \operatorname{Area}(\vec{A})$ indica que la clasificación de los títulos del docente coinciden con el área de las asignaturas, de acuerdo con la clasificación CINE (UNESCO, 2010)

- $\quad p_{1}, p_{2}, p_{3}$ son pesos que permiten ponderar el nivel de importancia de cada componente. La sumatoria de pesos debe ser igual a 1.

El componente de evaluación estudiantil se compone de una suma ponderada de las evaluaciones para todo par docente-asignatura de la consulta multiplicada por un factor de penalización. Los valores se calcularán siempre desde el ciclo actual hasta $N$ períodos lectivos hacia atrás, este factor se puede apreciar en la Figura 3:

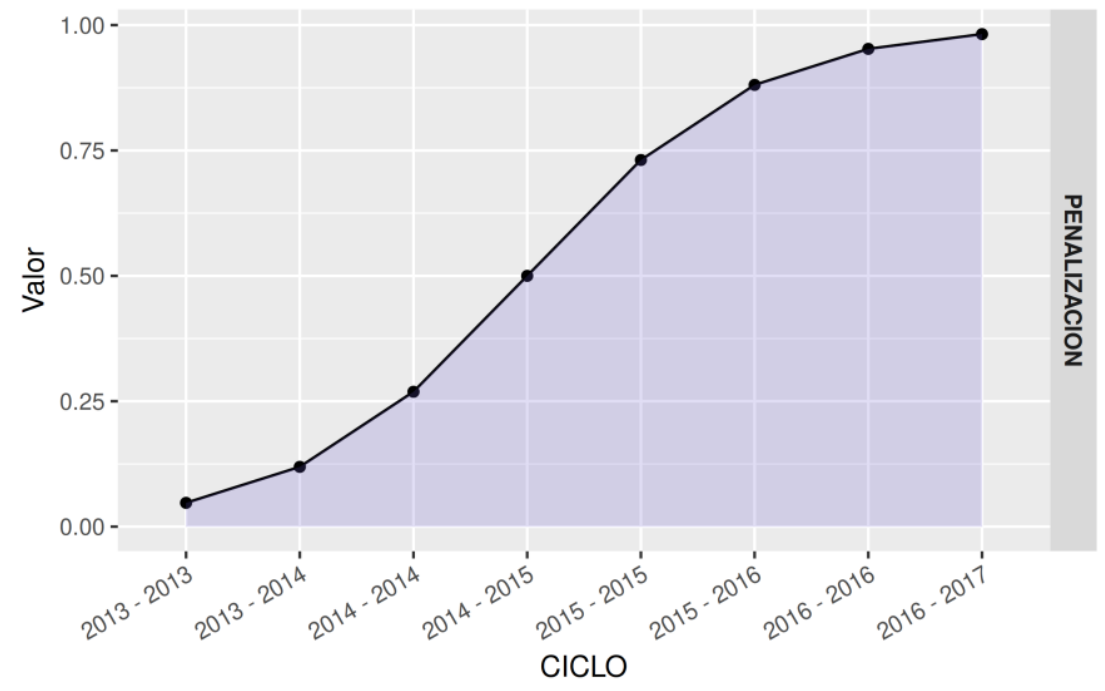

Figura 3. Factor de penalización

Dicha penalización pretende dar más relevancia en el cálculo de pertinencia a las asignaturas más recientes que haya dictado un docente D. Como muestra en la Figura 4, las calificaciones más antiguas pierden relevancia, no pueden ser superiores al factor de penalización. 


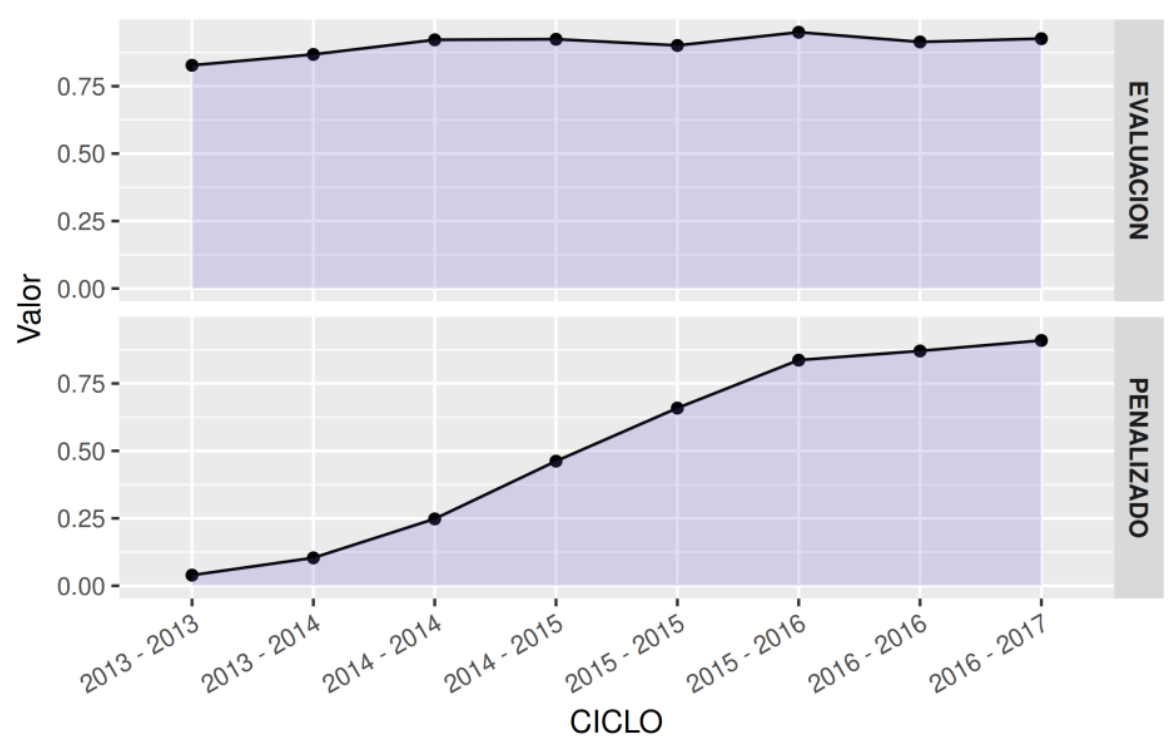

Figura 4.Ejemplo de pérdida de relevancia para un caso de evaluación docente

\subsection{Generación de horarios}

Se realizó la implementación de un algoritmo matemático mediante Álgebra Lineal y Programación Entera Lineal para la asignación automática mediante restricciones. Para este estudio se realizaron sendas entrevistas con el personal de la Universidad Politécnica Salesiana para poder llevar a cabo un análisis de los criterios que son tomados en cuenta al momento de diagramar un horario de clases.

Para nuestro estudio han sido consideradas las siguientes restricciones.

- Un período no puede repetirse en un mismo día para una materia, de esta manera no se asignan varias materias de un mismo ciclo a un mismo periodo de clases.

- Un docente no puede dar la misma materia en un mismo día.

- Evitar cruces de varias materias que dé un docente en un mismo período de clases.

- Garantizar que una materia no exceda el número de créditos en una semana de clases.

El modelo propuesto a utilizar es el modelo de Programación Lineal Entera. A continuación se presentará cada uno de los elementos matemáticos que conforman la propuesta.

\subsubsection{Función objetivo}

En virtud que se pretende maximizar los espacios utilizados por los docentes, emplearemos la función objetivo que se describe en la ecuación (2):

$$
\max \left(\sum_{p} \sum_{q} \sum_{r} \sum_{s} \sum_{t} X_{p, q, r, s, t} \cdot D_{p, q, s} \cdot \beta_{p, r}\right)
$$

Dónde:

- $\quad X_{\text {pqrst }}$ Variable de decisión binaria, indica que el docente fue ubicado en un día de la semana, en un período de clases en una materia perteneciente a un ciclo. 
- $\quad D_{p q s}$ es un parámetro de penalización, pues contiene la disponibilidad de un docente $(p)$ en un período $(s)$ en un día $(q)$. Por lo que en caso de asignar un docente en un período que no le corresponda, se obtendrá un valor de penalidad muy negativo para que la función objetivo que tiene por objeto el maximizar, pueda saber que no debe volver a asignar en un espacio equivocado. Si por el contrario la asignación es exitosa entonces el valor obtenido será 1.

Tabla 1. Ejemplo de la distribución de horas disponibles para dar clases de un docente. Estos datos se utilizan para el parámetro de penalización $D_{p q s}$

\begin{tabular}{|l|c|c|c|c|c|c|}
\hline $\begin{array}{l}\text { Docente } \\
\text { Día }\end{array}$ & Lunes & Martes & Miércoles & Jueves & Viernes & Sábado \\
\hline \multirow{4}{*}{ Docente 1 } & 1 & -10 & -10 & -10 & -10 & 1 \\
\cline { 2 - 7 } & 1 & -10 & -10 & -10 & -10 & 1 \\
\cline { 2 - 7 } & -10 & -10 & 1 & -10 & 1 & -10 \\
\cline { 2 - 7 } & -10 & -10 & 1 & -10 & 1 & -10 \\
\cline { 2 - 7 } & 1 & -10 & 1 & 1 & -10 & -10 \\
\cline { 2 - 7 } & 1 & -10 & 1 & 1 & -10 & -10 \\
\hline
\end{tabular}

$$
D_{p q s}=\left\{\begin{array}{cc}
1 & \text { Si el profesor tiene disponibilidad } \\
\text { para dictar clase en el período asignado } \\
-10 & \text { Penalización debido a asignación errónea }
\end{array}\right.
$$

- $\quad \beta_{p r}$ Ponderación obtenida de la API de Perfiles de Docentes.

Tabla 2. Ejemplo de ponderaciones $\beta_{p r}$. Este factor relaciona al docente con la materia.

\begin{tabular}{|c|c|c|}
\hline Docente & Ponderación & Materia \\
\hline Docente 1 & 0.9 & Materia 1 \\
\hline Docente 1 & 0.1 & Materia 2 \\
\hline
\end{tabular}

\subsubsection{Restricciones fuertes}

$$
\sum_{p} \sum_{q=a}^{b} \sum_{t} X_{p, q, r, s, t} \leq 1 ; \forall r, s
$$

- Esta restricción evita que un período se repita en un mismo día para una materia, de tal manera que no se asignen varias materias de un mismo ciclo a un mismo período de clases.

$$
\sum_{p} \sum_{q}^{b} \sum_{r} X_{p, q, r, s, t} \leq 1 ; \forall s, t
$$

- Evita que un docente de la misma materia más de una vez en el mismo día.

$$
\sum_{p} \sum_{q}^{b} \sum_{s} X_{p, q, r, s, t} \leq 1 ; \forall r, t
$$


- Evita cruces de varias materias que de un docente en un mismo período de clases.

$$
\sum_{t} X p, q, r, s, t=N_{r} ; \forall p, q, s, t
$$

- Garantiza que una materia no exceda el número de créditos en una semana de clases.

\section{Resultados}

La evaluación de los resultados se compone de dos etapas. Evaluamos primero la calidad de los pares docente-asignatura recomendados a base del análisis de históricos y desempeño docente y las consideraciones de similitud entre el perfil docente y las asignaturas de interés; a partir de dichos resultados se generaron varias distribuciones de horarios para los docentes y asignaturas seleccionados como mejores candidatos.

Las bases de datos utilizadas para esta propuesta tienen las siguientes consideraciones:

- Se emplearon 3 mil registros correspondientes a evaluaciones estudiantiles e históricos académicos.

- Se tomaron 133 mil registros acerca del perfil docente relacionado con la clasificación de las áreas de conocimiento correspondientes a su formación.

- La referencia de ciclos lectivos analizados hacia atrás (constante $N$ ) ha sido definida para 10 ciclos.

En la Figura 5 observamos resultados que arroja una consulta de los mejores pares docente-asignatura filtrados por asignatura para "Control de calidad", el valor de pertinencia se presenta escalado de 0 a 5 . Además, el sistema permite generar una representación visual de los datos de la consulta para poder brindar un enfoque más claro del porqué se asigna dicho valor de pertinencia en comparación con los demás docentes.

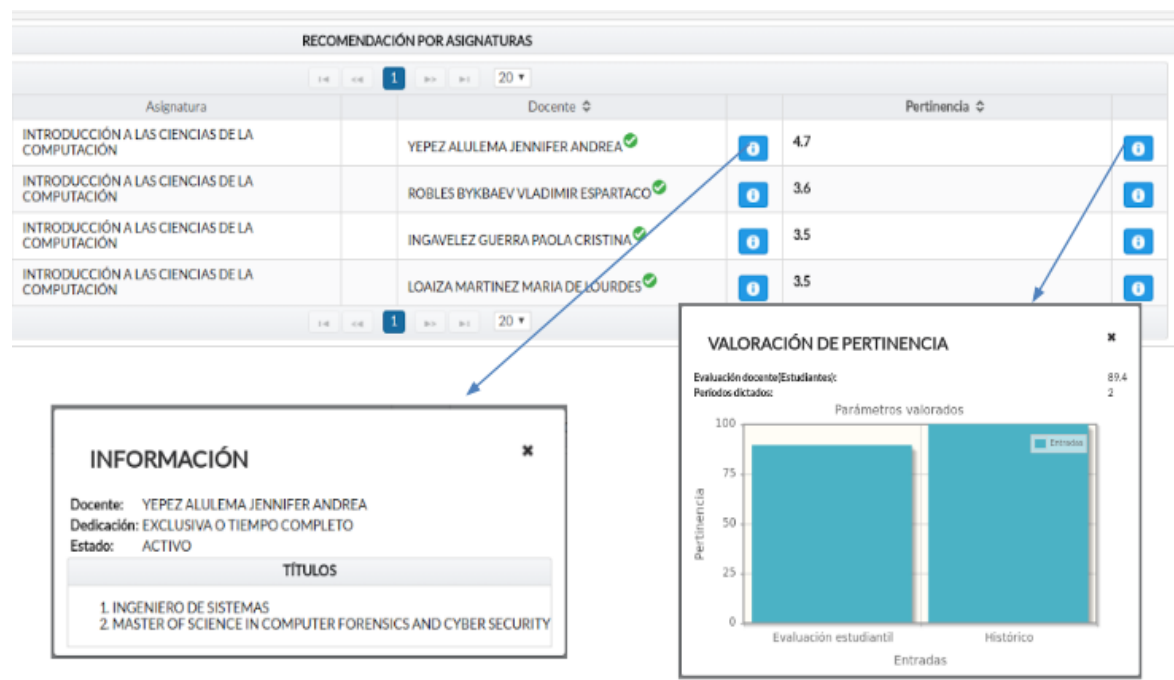

Figura 5.Ejemplo de resultados y reportes que genera el sistema en la generación de pares docente-asignatura.

A fin de evaluar las recomendaciones que ha sugerido el sistema, se realizó una valoración con 5 expertos del ámbito académico. Para ello se empleó una encuesta mediante la escala de Likert (Echauri, Minami y Sandoval, 2014) y se consideraron los 
siguientes criterios: pertinencia de la recomendación, coherencia y el rendimiento general del sistema. En la Tabla 3 se pueden apreciar las evaluaciones iniciales que obtuvo el sistema en los criterios, y como se observa, esta valoración inicial es muy positiva.

Tabla 3. Resultados preliminares de la evaluación del sistema con los directivos universitarios.

\begin{tabular}{cccc}
\hline & Pertinencia & Coherencia & Rendimiento \\
\hline & 4 & 3 & 5 \\
5 & 4 & 4 \\
& 3 & 4 & 4 \\
& 5 & 5 & 5 \\
& 4 & 4 & 3 \\
\hline Promedio & 4,2 & 4 & 4,2 \\
\hline
\end{tabular}

Asimismo, a fin de probar el algoritmo encargado de la generación de horarios, se implementó un script en Python, utilizando la librería PuLP (Mitchell, OSullivan, y Dunning, 2011) y el Solver GLPK (Makhorin, 2008). Con ello, se realizó un estudio piloto que empleó como corpus la información del primer ciclo y una materia de segundo ciclo de la carrera de Ingeniería en Computación. De esta manera se pudo verificar que el algoritmo matemático está correctamente planteado, pues tanto los docentes como las materias y los horarios evitaron cruces que vayan en contra de las restricciones. El resultado que genera el sistema se puede apreciar en la Figura 6.

\begin{tabular}{|c|c|c|c|c|}
\hline \multicolumn{5}{|c|}{ Horario de Clases } \\
\hline \multirow{2}{*}{ Día de Clases } & \multicolumn{2}{|c|}{ Semestre 1} & \multicolumn{2}{|c|}{ Semestre 2} \\
\hline & Clase & Docente & Clase & Docente \\
\hline Lunes 07:00-09:00 & Docente 1 & CALCULO DE UNA VARIABLE & & \\
\hline Lunes 09:00-11:00 & Docente 2 & ANTROPOLOGIA & & \\
\hline Lunes 11:00- 13:00 & Docente 3 & INTRODUCC. C. COMPUT. & & \\
\hline \multicolumn{5}{|l|}{ Lunes 13:00- 15:00 } \\
\hline Lunes 15:00-17:00 & Docente 4 & ALGEBRA LINEAL & & \\
\hline Lunes 17:00- 19:00 & Docente 4 & ALGEBRA LINEAL PR & & \\
\hline Lunes 19:00-21:00 & Docente 5 & CALCULO 1 VARIABLE PR & & \\
\hline Martes 07:00-09:00 & Docente 4 & ALGEBRA LINEAL PR & & \\
\hline \multicolumn{5}{|l|}{ Martes 09:00-11:00 } \\
\hline \multicolumn{5}{|l|}{ Martes 11:00-13:00 } \\
\hline Martes 13:00- 15:00 & Docente 3 & INTRODUCC. C. COMPUT. & & \\
\hline Martes 15:00-17:00 & Docente 4 & ALGEBRA LINEAL & & \\
\hline Martes 17:00- 19:00 & Docente 1 & COMUNICA. ORAL Y ES. & & \\
\hline \multicolumn{5}{|l|}{ Martes 19:00- 21:00 } \\
\hline \multicolumn{5}{|l|}{ Miércoles 07:00-09:00 } \\
\hline \multicolumn{5}{|l|}{ Miércoles 09:00-11:00 } \\
\hline Miércoles 11:00- 13:00 & Docente 6 & LOGICA & & \\
\hline Miércoles 13:00- 15:00 & Docente 5 & CALCULO 1 VARIABLE PR & & \\
\hline Miércoles 15:00-17:00 & & & Docente 6 & TEORIA CRITICA \\
\hline Miércoles 17:00- 19:00 & Docente 2 & ANTROPOLOGIA & & \\
\hline \multicolumn{5}{|l|}{ Miércoles 19:00- 21:00 } \\
\hline \multicolumn{5}{|l|}{ Jueves 07:00-09:00 } \\
\hline Jueves 09:00-11:00 & Docente 1 & COMUNICA. ORAL Y ES. & & \\
\hline \multicolumn{5}{|l|}{ Jueves 11:00-13:00 } \\
\hline Jueves 13:00- 15:00 & & & & TEORIA CRITICA \\
\hline Jueves 15:00-17:00 & & & Docente 6 & \\
\hline Jueves 17:00- 19:00 & Docente 6 & LOGICA & & \\
\hline \multicolumn{5}{|l|}{ Jueves 19:00-21:00 } \\
\hline Viernes 07:00-09:00 & Docente 1 & CALCULO DE UNA VARIABLE & & \\
\hline \multicolumn{5}{|l|}{ Viernes 09:00-11:00 } \\
\hline \multicolumn{5}{|l|}{ Viernes 11:00- 13:00 } \\
\hline \multicolumn{5}{|l|}{ Viernes 13:00- 15:00 } \\
\hline \multicolumn{5}{|l|}{ Viernes 15:00-17:00 } \\
\hline \multicolumn{5}{|l|}{ Viernes 17:00- 19:00 } \\
\hline Viernes 19:00- 21:00 & & & & \\
\hline
\end{tabular}

Figura 6. Ejemplo de un horario generado de forma automática para el primer semestre de la carrera de Ingeniería en Computación

A continuación se describe un ejemplo del proceso de cálculo que se realiza mediante la programación entera lineal durante la generación de los horarios. A fin de maximizar la función objetivo, se la presenta a continuación como la suma del producto 
de los elementos de la matriz por los elementos de penalización de horarios de los docentes:

$$
\begin{aligned}
& \text { Maximizar } \\
& \text { OBJ: } 0.9 x_{-}\left(0,{ }_{-} 0,{ }_{-} 0,{ }_{-} 0\right)+0.1 x_{-}\left(0,{ }_{-} 0,{ }_{-} 0, \_1\right)+0.7 x_{-}\left(0,{ }_{-} 0,{ }_{-} 0,{ }_{-} 2\right) \\
& +0.9 x_{-}\left(0,{ }_{-} 0, \_1,{ }_{-} 0\right)+0.1 x_{-}\left(0,{ }_{-} 0,{ }_{-} 1,{ }_{-} 1\right)+0.7 x_{-}\left(0,{ }_{-} 0,{ }_{-} 1,{ }_{-} 2\right)
\end{aligned}
$$

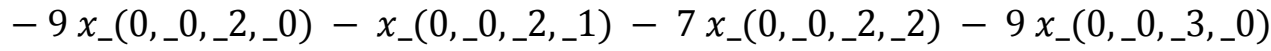

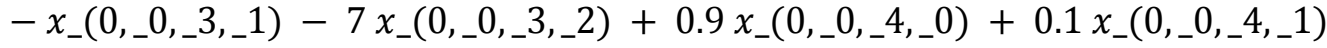

$$
\begin{aligned}
& +0.7 x_{-}\left(0,{ }_{-} 0,{ }_{-} 4,{ }_{-} 2\right)+0.9 x_{-}\left(0,{ }_{-} 0,{ }_{-} 5,{ }_{-} 0\right)+0.1 x_{-}\left(0,{ }_{-} 0,{ }_{-} 5,{ }_{-} 1\right) \\
& +0.7 x_{-}\left(0,{ }_{-} 0, \_5, \_2\right)+0.9 x_{-}\left(0,{ }_{-} 0,{ }_{-} 6, \_0\right)+0.1 x_{-}\left(0,{ }_{-} 0,{ }_{-} 6,-1\right)+ \\
& -5 x_{-}\left(2, \_5, \_6, \_1\right)-5 x_{-}\left(2, \_5, \_6, \_2\right)
\end{aligned}
$$

La función objetivo está sujeta a restricciones que permiten el ajuste del modelo matemático a las condiciones requeridas. En total se generaron 183 restricciones, las cuales vienen dadas por la combinación de los elementos binarios que conforman la matriz entera lineal sometida a las restricciones establecidas del modelo.

Cada una de las restricciones se presenta como se indica seguidamente:

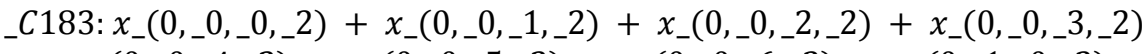

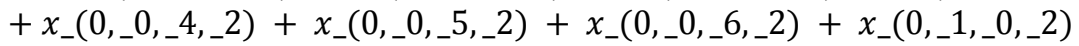

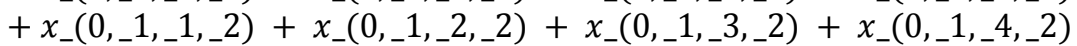

$$
\begin{aligned}
& +x_{-}\left(0,{ }_{-} 1,{ }_{-} 5, \_2\right)+x_{-}\left(0,{ }_{-} 1,{ }_{-} 6,{ }_{-} 2\right)+x_{-}\left(0,{ }_{-} 2,{ }_{-} 0,{ }_{-} 2\right)+x_{-}\left(0,{ }_{-} 2,{ }_{-} 1,{ }_{-} 2\right)
\end{aligned}
$$

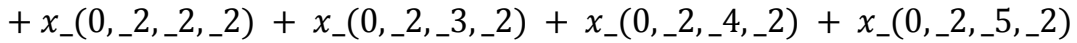

$$
\begin{aligned}
& +x_{-}\left(0, \_2,{ }_{-} 6, \_2\right)+x_{-}\left(0,{ }_{-} 3, x_{-} 0, \_2\right)+x_{-}\left(0, x_{-} 3,{ }_{-} 1,{ }_{-} 2\right)+x_{-}\left(0,{ }_{-} 3,{ }_{-} 2,{ }_{-} 2\right)
\end{aligned}
$$

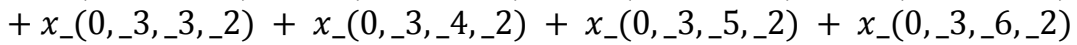

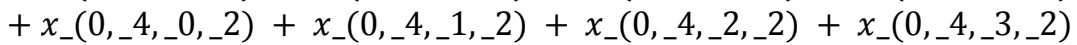

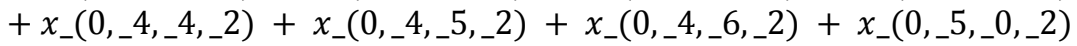

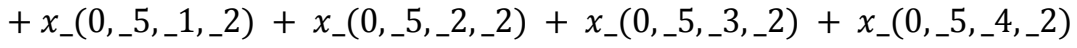

$$
\begin{aligned}
& +x_{-}\left(0, \_5,{ }_{-} 5, \_2\right)+x_{-}\left(0,{ }_{-} 5,{ }_{-} 6,{ }_{-} 2\right)+x_{-}\left(1,{ }_{-} 0,{ }_{-} 0, \_2\right)+x_{-}\left(1,{ }_{-} 0,{ }_{-} 1,{ }_{-} 2\right)
\end{aligned}
$$

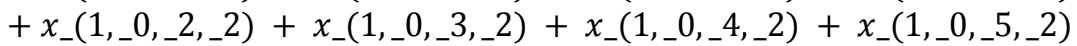

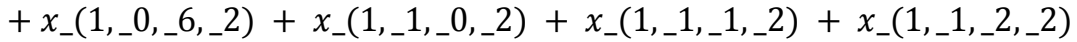

$$
\begin{aligned}
& +x_{-}\left(1,1_{-} 1,{ }_{-} 3, \_2\right)+x_{-}\left(1,{ }_{-} 1, x_{-} 4, x_{-} 2\right)+x_{-}\left(1,1_{-} 1,{ }_{-} 5, \_2\right)+x_{-}\left(1,1_{-} 1,{ }_{-} 6,{ }_{-} 2\right) \\
& +x_{-}\left(1, \_2,0_{-} 0, \_2\right)+x_{-}\left(1, \_2,1_{-} 1, \_2\right)+x_{-}\left(1, \_2, \_2, \_2\right)+x_{-}\left(1,{ }_{-} 2,{ }_{-} 3,{ }_{-} 2\right)
\end{aligned}
$$

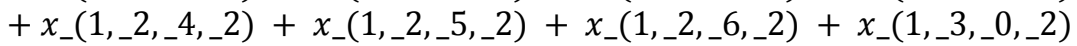

$$
\begin{aligned}
& +x_{-}\left(1, \_3, \_1, \_2\right)+x_{-}\left(1,{ }_{-} 3,{ }_{-} 2, \_2\right)+x_{-}\left(1, x_{-} 3, \_3, \_2\right)+x_{-}\left(1,{ }_{-} 3,{ }_{-} 4, \_2\right)
\end{aligned}
$$

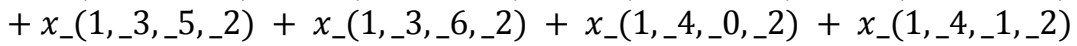

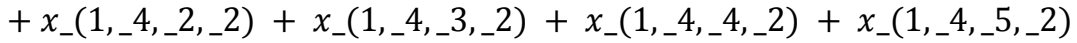

$$
\begin{aligned}
& +x_{-}\left(1,1_{-} 4,{ }_{-} 6, \_2\right)+x_{-}\left(1,{ }_{-} 5,{ }_{-} 0, x_{-} 2\right)+x_{-}\left(1,{ }_{-} 5,{ }_{-} 1,{ }_{-} 2\right)+x_{-}\left(1,1_{-} 5,{ }_{-} 2,{ }_{-} 2\right) \\
& +x_{-}\left(1,{ }_{-} 5,{ }_{-} 3,{ }_{-} 2\right)+x_{-}\left(1,{ }_{-} 5,{ }_{-} 4, x_{-} 2\right)+x_{-}\left(1,{ }_{-} 5,{ }_{-} 5, \_2\right)+x_{-}\left(1,{ }_{-} 5,{ }_{-} 6,{ }_{-} 2\right)
\end{aligned}
$$

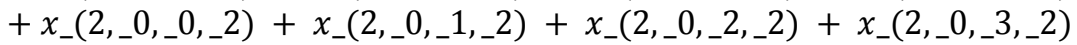

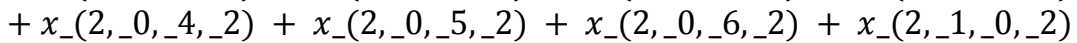

$$
\begin{aligned}
& +x_{-}\left(2,,_{-} 1,1_{-}, 2\right)+x_{-}\left(2,{ }_{-} 1,{ }_{-} 2,{ }_{-} 2\right)+x_{-}\left(2,{ }_{-} 1,{ }_{-} 3,{ }_{-} 2\right)+x_{-}\left(2,{ }_{-} 1,{ }_{-} 4,{ }_{-} 2\right) \\
& +x_{-}\left(2,{ }_{-} 1,{ }_{-} 5, \_2\right)+x_{-}\left(2, x_{-} 1, x_{-} 6, x_{-} 2\right)+x_{-}\left(2,{ }_{-} 2,{ }_{-} 0,{ }_{-} 2\right)+x_{-}\left(2,{ }_{-} 2,{ }_{-} 1,{ }_{-} 2\right) \\
& +x_{-}\left(2,{ }_{-} 2,{ }_{-} 2,{ }_{-} 2\right)+x_{-}\left(2, x_{-} 2,{ }_{-} 3,{ }_{-} 2\right)+x_{-}\left(2,{ }_{-} 2,{ }_{-} 4,{ }_{-} 2\right)+x_{-}\left(2,{ }_{-} 2,{ }_{-} 5,{ }_{-} 2\right) \\
& +x_{-}\left(2, \_2,{ }_{-} 6, \_2\right)+x_{-}\left(2,{ }_{-} 3,{ }_{-} 0, \_2\right)+x_{-}\left(2,{ }_{-} 3,{ }_{-} 1,{ }_{-} 2\right)+x_{-}\left(2,{ }_{-} 3,{ }_{-} 2,{ }_{-} 2\right) \\
& +x_{-}\left(2,{ }_{-} 3,{ }_{-} 3, \_2\right)+x_{-}\left(2,{ }_{-} 3, x_{-} 4, x_{-} 2\right)+x_{-}\left(2,{ }_{-} 3,{ }_{-} 5,{ }_{-} 2\right)+x_{-}\left(2,{ }_{-} 3,{ }_{-} 6,-2\right)
\end{aligned}
$$

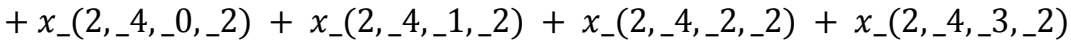

$$
\begin{aligned}
& +x_{-}\left(2,{ }_{-} 4,{ }_{-} 4,{ }_{-} 2\right)+x_{-}\left(2,{ }_{-} 4,{ }_{-} 5,{ }_{-} 2\right)+x_{-}\left(2,{ }_{-} 4,{ }_{-} 6,{ }_{-} 2\right)+x_{-}\left(2,{ }_{-} 5,{ }_{-} 0,{ }_{-} 2\right) \\
& +x_{-}\left(2,{ }_{-} 5,{ }_{-} 1, \_2\right)+x_{-}\left(2,{ }_{-} 5,{ }_{-} 2,{ }_{-} 2\right)+x_{-}\left(2,{ }_{-} 5,{ }_{-} 3,{ }_{-} 2\right)+x_{-}\left(2,{ }_{-} 5,{ }_{-} 4,{ }_{-} 2\right) \\
& +x_{-}\left(2,{ }_{-} 5, \_5, \_2\right)+x_{-}\left(2, \_5, \_6, \_2\right)=6
\end{aligned}
$$

\section{Discusión}

La generación de horarios es un tema con múltiples soluciones, la optimización en la construcción de horarios trata de encontrar las mejores distribuciones de asignaciones docentes, las aproximaciones relacionadas por lo general pretenden optimizar conjuntos 
de reglas para encontrar la mejor solución posible; entre estas soluciones podemos encontrar técnicas basadas en satisfacibilidad proposicional, reglas basadas en currículos institucionales, para optimizar asignaciones de cursos que comparten varios estudiantes, algoritmos genéticos, entre otras.

La propuesta presentada tiene un enfoque que, a más de encontrar soluciones a conjuntos de reglas, pretende que los docentes que se asignan tengan la mejor experiencia y formación profesional sobre la asignatura entre los docentes disponibles, a medida de lo posible. De esta manera se evidencia no solo la optimización de reglas sino el aumento del desempeño y calidad de docencia, aumentando el rendimiento académico general del estudiantado.

\section{Conclusiones y recomendaciones}

En este artículo se ha presentado una propuesta inicial de un sistema experto para la asignación de materias a docentes y la generación de horarios en el ámbito universitario. Como se ha podido apreciar, la propuesta inicial permite realizar la asignación de forma óptima a pesar que se cuente con grandes cantidades de registros tanto de asignaturas como de docentes.

Los métodos matemáticos empleados son bastante eficientes, ya que el consumo de recursos es mínimo (si tomamos en cuenta que las restricciones deben estar correctamente planteadas). De igual forma, las técnicas de minería de datos pueden ser remplazadas o enriquecidas con algoritmos de aprendizaje de máquina (redes neuronales y de creencia profunda, árboles de decisión, etc.).

A pesar de lo expuesto, consideramos oportuno señalar que el presente trabajo presenta algunas limitaciones tanto para la asignación de materias como para la generación de horarios. Principalmente, es fundamental considerar aspectos relacionados con la logística de los espacios físicos de estudio, así como de los materiales requeridos en determinadas asignaturas (física, redes inalámbricas, etc).

Como líneas de trabajo futuro se plantean las siguientes:

- Desarrolla de un módulo de análisis de restricciones basado en algoritmos genéticos, a fin de analizar las ventajas y desventajas de esta técnica en la generación de horarios.

- Desarrollar un módulo que permita extraer información de la producción científica de los docentes, a fin de incluir un eje más que permita enriquecer el perfil docente desde el punto de vista de la investigación.

\section{Bibliografía}

CES. (2010). Ley Orgánica de Educación Superior, República del Ecuador. Consejo de Educación Superior

Echauri, A. M. F., Minami, H., \& Sandoval, M. J. I. (2014). "La Escala de Likert en la evaluación docente: acercamiento a sus características y principios metodológicos". Perspectivas Docentes, (50).

Garbanzo Vargas, G. M. (2007). "Factores asociados al rendimiento académico en estudiantes universitarios, una reflexión desde la calidad de la educación superior pública”. Educación, 31 (1).

Larrea, E., y Granados, V. (2016). "El sistema de educación superior para la sociedad del buen vivir basada en el conocimiento". Guayaquil: Universidad Católica de Santiago de Guayaquil.

Makhorin, A. (2008). GLPK (GNU linear programming kit). http://www. gnu. org/software/glpk/.

Mitchell, S., OSullivan, M., \& Dunning, I. (2011). "PuLP: a linear programming toolkit for python. The University of Auckland, Auckland, New Zealand".

Pesántez-Avilés, F., Calle-López, D., Robles-Bykbaev, V., Rodas-Tobar, M., y Vásquez- 
Vásquez, C. (2017). "A recommender system based on data mining techniques to support the automatic assignment of courses to teachers in higher education". En Inciscos 2017: Proceedings of the 2nd international conference on information systems and computer science.

Sánchez Fleitas, N., Comas Rodríguez, R., García Lorenzo, M., y Riverol Quesada, A. (2016). "Modelo de manejo de datos, con el uso de inteligencia artificial, para un sistema de información geográfica en el sector energético". Enfoque UTE, 7 (3).

UNESCO. (2013). "Clasificación Internacional Normalizada de la Educación-CINE".

Zabalza, M. Á., y Beraza, M. Á. Z. (2003). Competencias docentes del profesorado universitario: calidad y desarrollo profesional (Vol. 4). Narcea Ediciones.

\section{Anexo 1.}

Función Objetivo para generación automatizada de horarios.

$$
\begin{aligned}
& \text { Maximizar Función Objetivo } \\
& O B J: 0.9 x_{-}\left(0,,_{-} 0,{ }_{-} 0,{ }_{-} 0\right)+0.1 x_{-}\left(0,,_{-} 0,{ }_{-} 0, \_1\right)+0.7 x_{-}\left(0,,_{-} 0,,_{-} 0,{ }_{-} 2\right) \\
& +0.9 x_{-}\left(0,{ }_{-} 0,{ }_{-} 1,{ }_{-} 0\right)+0.1 x_{-}\left(0,{ }_{-} 0,{ }_{-} 1,{ }_{-} 1\right)+0.7 x_{-}\left(0,{ }_{-} 0,{ }_{-} 1,{ }_{-} 2\right) \\
& -9 x_{-}\left(0, \_0, \_2, \_0\right)-x_{-}\left(0,{ }_{-} 0, \_2, \_1\right)-7 x_{-}\left(0,{ }_{-} 0,{ }_{-} 2, \_2\right)-9 x_{-}\left(0,{ }_{-} 0,{ }_{-} 3,{ }_{-} 0\right)
\end{aligned}
$$

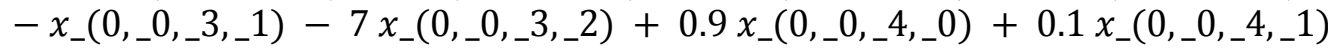

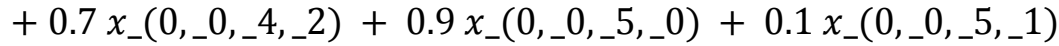

$$
\begin{aligned}
& +0.7 x_{-}\left(0,{ }_{-} 0,{ }_{-} 5, \_2\right)+0.9 x_{-}\left(0,{ }_{-} 0,{ }_{-} 6,,_{-} 0\right)+0.1 x_{-}\left(0,{ }_{-} 0,{ }_{-} 6,{ }_{-} 1\right)
\end{aligned}
$$

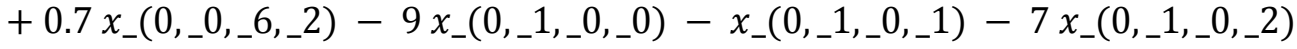

$$
\begin{aligned}
& -9 x_{-}\left(0,{ }_{-} 1, \_1, \_0\right)-x_{-}\left(0,{ }_{-} 1, \_1,{ }_{-} 1\right)-7 x_{-}\left(0,{ }_{-} 1,{ }_{-} 1, \_2\right)-9 x_{-}\left(0,{ }_{-} 1, x_{-} 2,{ }_{-} 0\right) \\
& -x_{-}\left(0, \_1, \_2, \_1\right)-7 x_{-}\left(0,{ }_{-} 1, \_2, \_2\right)-9 x_{-}\left(0, \_1, \_3, \_0\right)-x_{-}\left(0,{ }_{-} 1, \_3, \_1\right) \\
& -7 x_{-}\left(0,{ }_{-} 1,{ }_{-} 3, \_2\right)-9 x_{-}\left(0, \_1,{ }_{-} 4, x_{-} 0\right)-x_{-}\left(0,{ }_{-} 1, x_{-} 4, \_1\right)-7 x_{-}\left(0, x_{-} 1,{ }_{-} 4, x_{-} 2\right) \\
& -9 x_{-}\left(0,{ }_{-} 1,{ }_{-} 5, \_0\right)-x_{-}\left(0,{ }_{-} 1, \_5, \_1\right)-7 x_{-}\left(0,{ }_{-} 1, \_5, \_2\right)-9 x_{-}\left(0, \_1, x_{-} 6, x_{-} 0\right)
\end{aligned}
$$

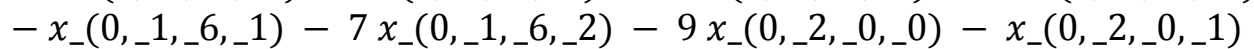

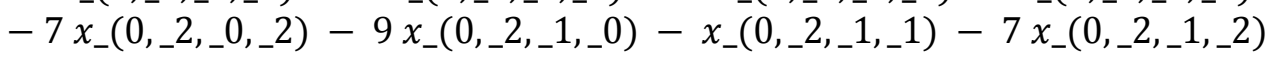

$$
\begin{aligned}
& +0.9 x_{-}\left(0,{ }_{-} 2, \_2,{ }_{-} 0\right)+0.1 x_{-}\left(0,{ }_{-} 2,{ }_{-} 2, \_1\right)+0.7 x_{-}\left(0,{ }_{-} 2,{ }_{-} 2,{ }_{-} 2\right) \\
& +0.9 x_{-}\left(0, \_2, \_3, \_0\right)+0.1 x_{-}\left(0,{ }_{-} 2,{ }_{-} 3, \_1\right)+0.7 x_{-}\left(0,{ }_{-} 2,{ }_{-} 3, \_2\right) \\
& +0.9 x_{-}\left(0,{ }_{-} 2,{ }_{-} 4,{ }_{-} 0\right)+0.1 x_{-}\left(0,{ }_{-} 2,{ }_{-} 4,{ }_{-} 1\right)+0.7 x_{-}\left(0,{ }_{-} 2,{ }_{-} 4,{ }_{-} 2\right) \\
& +0.9 x_{-}\left(0,{ }_{-} 2,{ }_{-} 5,{ }_{-} 0\right)+0.1 x_{-}\left(0,{ }_{-} 2, \_5, \_1\right)+0.7 x_{-}\left(0,{ }_{-} 2,{ }_{-} 5, \_2\right) \\
& +0.9 x_{-}\left(0,{ }_{-} 2,{ }_{-} 6,{ }_{-} 0\right)+0.1 x_{-}\left(0,{ }_{-} 2,{ }_{-} 6, \_1\right)+0.7 x_{-}\left(0, x_{-} 2, x_{-} 6, \_2\right)
\end{aligned}
$$

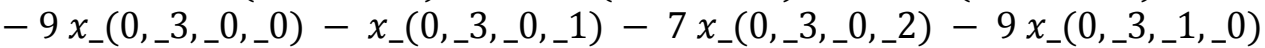

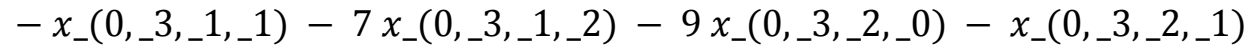

$$
\begin{aligned}
& -7 x_{-}\left(0,{ }_{-} 3, \_2, \_2\right)-9 x_{-}\left(0, \_3, \_3, \_0\right)-x_{-}\left(0,{ }_{-} 3, \_3, \_1\right)-7 x_{-}\left(0, \_3, \_3,22\right)
\end{aligned}
$$

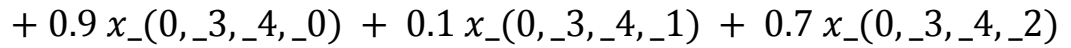

$$
\begin{aligned}
& +0.9 x_{-}\left(0,{ }_{-} 3,{ }_{-} 5,{ }_{-} 0\right)+0.1 x_{-}\left(0,{ }_{-} 3,{ }_{-} 5,{ }_{-} 1\right)+0.7 x_{-}\left(0,{ }_{-} 3,{ }_{-} 5, \ldots 2\right) \\
& +0.9 x_{-}\left(0,{ }_{-} 3,{ }_{-} 6,{ }_{-} 0\right)+0.1 x_{-}\left(0,{ }_{-} 3,{ }_{-} 6,{ }_{-} 1\right)+0.7 x_{-}\left(0,{ }_{-} 3,{ }_{-} 6,{ }_{-} 2\right) \\
& -9 x_{-}\left(0,{ }_{-} 4,{ }_{-} 0, \_0\right)-x_{-}\left(0,{ }_{-} 4,{ }_{-} 0,{ }_{-} 1\right)-7 x_{-}\left(0,{ }_{-} 4,{ }_{-} 0, \_2\right)-9 x_{-}\left(0,{ }_{-} 4,{ }_{-} 1,{ }_{-} 0\right)
\end{aligned}
$$

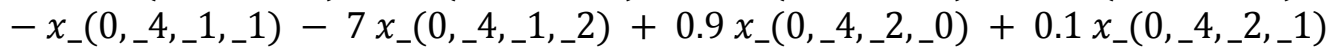

$$
\begin{aligned}
& +0.7 x_{-}\left(0,{ }_{-} 4, \_2,{ }_{-} 2\right)+0.9 x_{-}\left(0,{ }_{-} 4,{ }_{-} 3,{ }_{-} 0\right)+0.1 x_{-}\left(0,{ }_{-} 4, x_{-} 3, \_1\right) \\
& +0.7 x_{-}\left(0,{ }_{-} 4, \_3, \_2\right)-9 x_{-}\left(0,{ }_{-} 4, \_4, \_0\right)-x_{-}\left(0,{ }_{-} 4,{ }_{-} 4, \_1\right)-7 x_{-}\left(0,{ }_{-} 4,{ }_{-} 4,{ }_{-} 2\right) \\
& -9 x_{-}\left(0,{ }_{-} 4, \_5, \_0\right)-x_{-}\left(0,{ }_{-} 4, \_5, \_1\right)-7 x_{-}\left(0,{ }_{-} 4, \_5, \_2\right)-9 x_{-}\left(0,{ }_{-} 4,{ }_{-} 6,{ }_{-} 0\right) \\
& -x_{-}\left(0,{ }_{-} 4, \_6, \_1\right)-7 x_{-}\left(0,{ }_{-} 4, \_6,{ }_{-} 2\right)+0.9 x_{-}\left(0,{ }_{-} 5,{ }_{-} 0,{ }_{-} 0\right)+0.1 x_{-}\left(0,{ }_{-} 5,{ }_{-} 0, \_1\right) \\
& +0.7 x_{-}\left(0,{ }_{-} 5,{ }_{-} 0,{ }_{-} 2\right)+0.9 x_{-}\left(0,{ }_{-} 5,{ }_{-} 1,{ }_{-} 0\right)+0.1 x_{-}\left(0,{ }_{-} 5,{ }_{-} 1,{ }_{-} 1\right) \\
& +0.7 x_{-}\left(0,{ }_{-} 5, \_1, \_2\right)-9 x_{-}\left(0,{ }_{-} 5, \_2, \_0\right)-x_{-}\left(0, \_5, \_2, \_1\right)-7 x_{-}\left(0, \_5,{ }_{-} 2, \_2\right)
\end{aligned}
$$

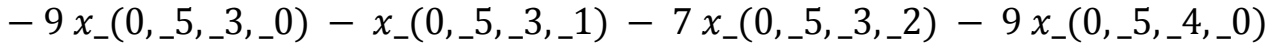

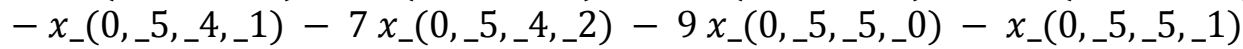

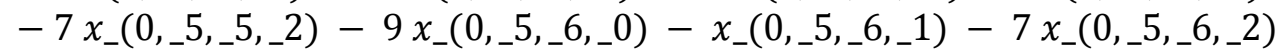

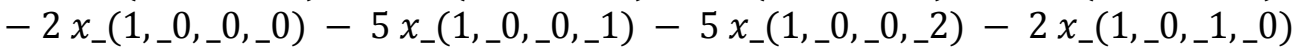

$$
\begin{aligned}
& -5 x_{-}\left(1,0_{-} 0, \_1,-1\right)-5 x_{-}\left(1,{ }_{-} 0, \_1, \_2\right)+0.2 x_{-}\left(1,{ }_{-} 0,{ }_{-} 2,{ }_{-} 0\right) \\
& +0.5 x_{-}\left(1, \_0, \_2, \_1\right)+0.5 x_{-}\left(1,{ }_{-} 0,{ }_{-} 2, \_2\right)+0.2 x_{-}\left(1,{ }_{-} 0, \_3, \_0\right)
\end{aligned}
$$




$$
\begin{aligned}
& +0.5 x_{-}\left(1,{ }_{-} 0,{ }_{-} 3, \_1\right)+0.5 x_{-}\left(1,{ }_{-} 0,{ }_{-} 3,{ }_{-} 2\right)-2 x_{-}\left(1,{ }_{-} 0,{ }_{-} 4,{ }_{-} 0\right)
\end{aligned}
$$

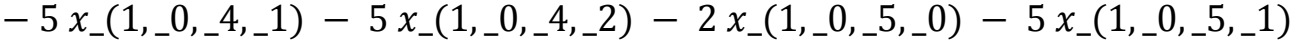

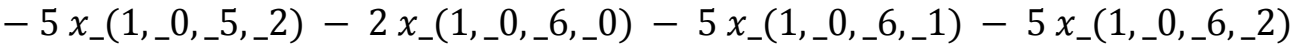

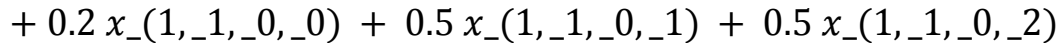

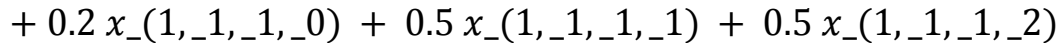

$$
\begin{aligned}
& +0.2 x_{-}\left(1, \_1, \_2, \_0\right)+0.5 x_{-}\left(1, \_1, \_2, \_1\right)+0.5 x_{-}\left(1, \_1, \_2, \_2\right) \\
& +0.2 x_{-}\left(1, \_1, \_3, \_0\right)+0.5 x_{-}\left(1, \_1, \_3, \_1\right)+0.5 x_{-}\left(1,1_{-} 1, \_3, \_2\right) \\
& +0.2 x_{-}\left(1, \_1,{ }_{-} 4, \_0\right)+0.5 x_{-}\left(1,{ }_{-} 1, \_4, \_1\right)+0.5 x_{-}\left(1,{ }_{-} 1,{ }_{-} 4,{ }_{-} 2\right) \\
& +0.2 x_{-}\left(1, \_1, \_5, \_0\right)+0.5 x_{-}\left(1,1_{-} 1, \_5, \_1\right)+0.5 x_{-}\left(1,1_{-} 1,{ }_{-} 5,{ }_{-} 2\right) \\
& +0.2 x_{-}\left(1, \_1, \_6,,_{-} 0\right)+0.5 x_{-}\left(1, \_1,{ }_{-} 6, \_1\right)+0.5 x_{-}\left(1,{ }_{-} 1,{ }_{-} 6,{ }_{-} 2\right) \\
& +0.2 x_{-}\left(1, \_2, \_0, \_0\right)+0.5 x_{-}\left(1, \_2, \_0, \_1\right)+0.5 x_{-}\left(1, \_2, \_0, \_2\right) \\
& +0.2 x_{-}\left(1, \_2,{ }_{-} 1,0\right)+0.5 x_{-}\left(1, \_2, \_1,-1\right)+0.5 x_{-}\left(1,{ }_{-} 2,{ }_{-} 1, \_2\right) \\
& -2 x_{-}\left(1, \_2, \_2,-0\right)-5 x_{-}\left(1, \_2, \_2, \_1\right)-5 x_{-}\left(1, \_2, \_2, \_2\right)-2 x_{-}\left(1, \_2, \_3, \_0\right) \\
& -5 x_{-}\left(1, \_2, \_3,-1\right)-5 x_{-}\left(1, \_2, \_3, \_2\right)-2 x_{-}\left(1, \_2, \_4, \_0\right)-5 x_{-}\left(1, \_2, \_4, \_1\right) \\
& -5 x_{-}\left(1, \_2,-4, \_2\right)-2 x_{-}\left(1, \_2, \_5, \_0\right)-5 x_{-}\left(1, \_2, \_5, \_1\right)-5 x_{-}\left(1, \_2, \_5, \_2\right)
\end{aligned}
$$

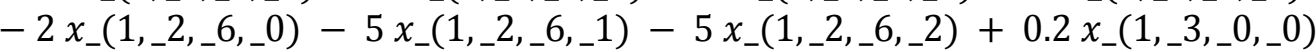

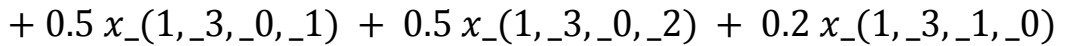

$$
\begin{aligned}
& +0.5 x_{-}\left(1,{ }_{-} 3, \_1,1\right)+0.5 x_{-}\left(1,{ }_{-} 3,{ }_{-} 1,{ }_{-} 2\right)+0.2 x_{-}\left(1,{ }_{-} 3,{ }_{-} 2,{ }_{-} 0\right)
\end{aligned}
$$

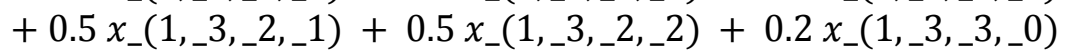

$$
\begin{aligned}
& +0.5 x_{-}\left(1,{ }_{-} 3,{ }_{-} 3,{ }_{-} 1\right)+0.5 x_{-}\left(1,{ }_{-} 3,{ }_{-} 3, x_{-} 2\right)-2 x_{-}\left(1,{ }_{-} 3,{ }_{-} 4,{ }_{-} 0\right)
\end{aligned}
$$

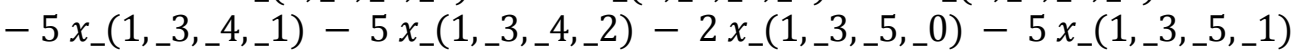

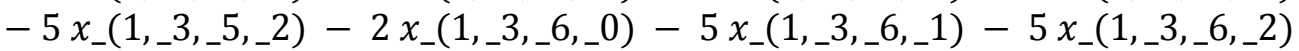

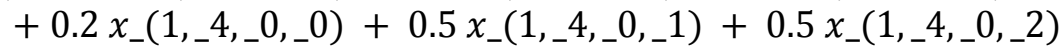

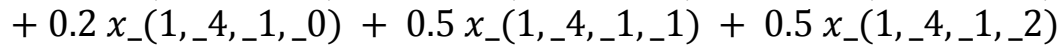

$$
\begin{aligned}
& -2 x_{-}\left(1, \_4, \_2, \_0\right)-5 x_{-}\left(1, \_4, \_2, \_1\right)-5 x_{-}\left(1,{ }_{-} 4, \_2,{ }_{-} 2\right)-2 x_{-}\left(1,{ }_{-} 4,{ }_{-} 3,{ }_{-} 0\right) \\
& -5 x_{-}\left(1, \_4, \_3, \_1\right)-5 x_{-}\left(1,1_{-} 4, \_3, \_2\right)+0.2 x_{-}\left(1,{ }_{-} 4,{ }_{-} 4,{ }_{-} 0\right)
\end{aligned}
$$

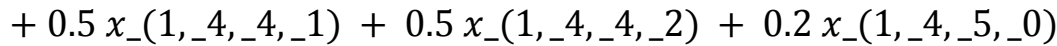

$$
\begin{aligned}
& +0.5 x_{-}\left(1,{ }_{-} 4, \_5, \_1\right)+0.5 x_{-}\left(1,{ }_{-} 4, \_5, \_2\right)+0.2 x_{-}\left(1,{ }_{-} 4,{ }_{-} 6,{ }_{-} 0\right)
\end{aligned}
$$

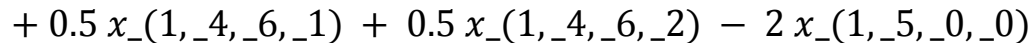

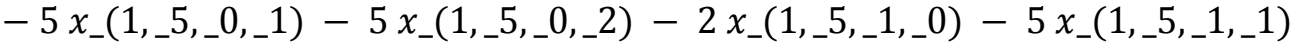

$$
\begin{aligned}
& -5 x_{-}\left(1, \_5, \_1, \_2\right)-2 x_{-}\left(1, \_5, \_2, \_0\right)-5 x_{-}\left(1, \_5, \_2,-1\right)-5 x_{-}\left(1, \_5, \_2, \_2\right)
\end{aligned}
$$

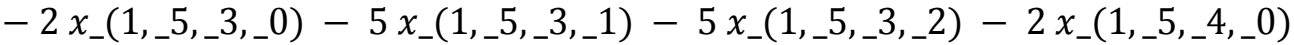

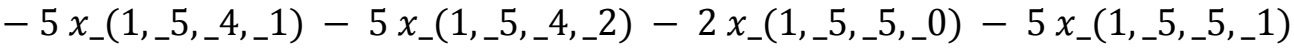

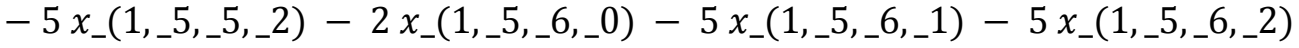

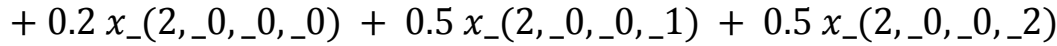

$$
\begin{aligned}
& +0.2 x_{-}\left(2,,_{-} 0,{ }_{-} 1,0\right)+0.5 x_{-}\left(2,{ }_{-} 0,{ }_{-} 1,{ }_{-} 1\right)+0.5 x_{-}\left(2,{ }_{-} 0,{ }_{-} 1,{ }_{-} 2\right) \\
& -2 x_{-}\left(2, \_0, \_2, \_0\right)-5 x_{-}\left(2, \_0, \_2, \_1\right)-5 x_{-}\left(2,{ }_{-} 0, \_2,-2\right)-2 x_{-}\left(2, \_0, \_3, \_0\right)
\end{aligned}
$$

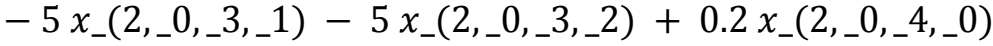

$$
\begin{aligned}
& +0.5 x_{-}\left(2,{ }_{-} 0,{ }_{-} 4,{ }_{-} 1\right)+0.5 x_{-}\left(2,{ }_{-} 0,{ }_{-} 4,{ }_{-} 2\right)+0.2 x_{-}\left(2,{ }_{-} 0,{ }_{-} 5,{ }_{-} 0\right)
\end{aligned}
$$

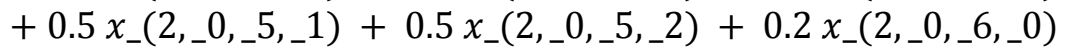

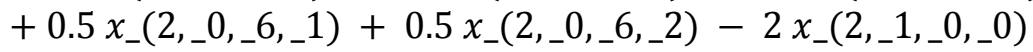

$$
\begin{aligned}
& -5 x_{-}\left(2,{ }_{-} 1,{ }_{-} 0,-1\right)-5 x_{-}\left(2,{ }_{-} 1,{ }_{-} 0,-2\right)-2 x_{-}\left(2,{ }_{-} 1, \_1,-0\right)-5 x_{-}\left(2,{ }_{-} 1,{ }_{-} 1,-1\right)
\end{aligned}
$$

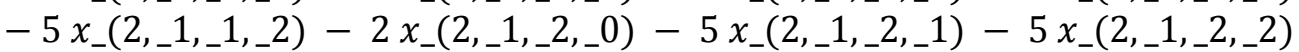

$$
\begin{aligned}
& -2 x_{-}\left(2, c_{-} 1,3,{ }_{-} 0\right)-5 x_{-}\left(2,{ }_{-} 1,3, \_1\right)-5 x_{-}\left(2,{ }_{-} 1,3,{ }_{-} 2\right)-2 x_{-}\left(2,{ }_{-} 1,4,{ }_{-} 0\right)
\end{aligned}
$$

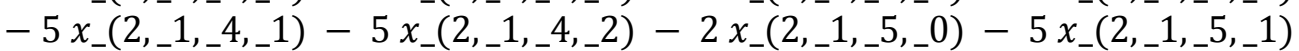

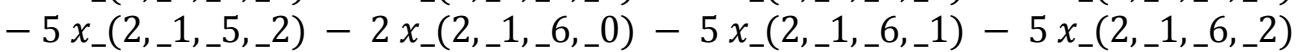

$$
\begin{aligned}
& -2 x_{-}\left(2, \_2,0,-0\right)-5 x_{-}\left(2, \_2, \_0, \_1\right)-5 x_{-}\left(2, \_2, \_0, \_2\right)-2 x_{-}\left(2, \_2, \_1, \_0\right) \\
& -5 x_{-}\left(2, \_2, \_1, \_1\right)-5 x_{-}\left(2,{ }_{-} 2, \_1, \_2\right)+0.2 x_{-}\left(2, \_2,22,0\right) \\
& +0.5 x_{-}\left(2, \_2, \_2, \_1\right)+0.5 x_{-}\left(2, \_2, \_2, \_2\right)+0.2 x_{-}\left(2, \_2, \_3, \_0\right) \\
& +0.5 x_{-}\left(2, \_2, \_3, \_1\right)+0.5 x_{-}\left(2, \_2, \_3, \_2\right)+0.2 x_{-}\left(2, \_2, \_4, \_0\right) \\
& +0.5 x_{-}\left(2, \_2, \_4, \_1\right)+0.5 x_{-}\left(2, \_2, \_4, \_2\right)+0.2 x_{-}\left(2, \_2, \_5, \_0\right) \\
& +0.5 x_{-}\left(2, \_2, \_5, \_1\right)+0.5 x_{-}\left(2, \_2, \_5, \_2\right)+0.2 x_{-}\left(2, \_2, \_6, \_0\right) \\
& +0.5 x_{-}\left(2, \_2, \_6, \_1\right)+0.5 x_{-}\left(2, \_2, \_6, \_2\right)-2 x_{-}\left(2,{ }_{-} 3,,_{-} 0,{ }_{-} 0\right)
\end{aligned}
$$




$$
\begin{aligned}
& -5 x_{-}\left(2, \_3, \_0, \_1\right)-5 x_{-}\left(2, \_3, \_0, \_2\right)-2 x_{-}\left(2, \_3, \_1,-0\right)-5 x_{-}\left(2, \_3, \_1,-1\right) \\
& -5 x_{-}\left(2, \_3, \_1, \_2\right)-2 x_{-}\left(2, \_3, \_2, \_0\right)-5 x_{-}\left(2, \_3, \_2, \_1\right)-5 x_{-}\left(2, \_3, \_2, \_2\right) \\
& -2 x_{-}\left(2, \_3,{ }_{-} 3, \_0\right)-5 x_{-}\left(2, \_3, \_3, \_1\right)-5 x_{-}\left(2, \_3,{ }_{-} 3, \_2\right)+0.2 x_{-}\left(2,{ }_{-} 3,{ }_{-} 4,{ }_{-} 0\right) \\
& +0.5 x_{-}\left(2,{ }_{-} 3,{ }_{-} 4,{ }_{-} 1\right)+0.5 x_{-}\left(2,{ }_{-} 3,{ }_{-} 4,{ }_{-} 2\right)+0.2 x_{-}\left(2,{ }_{-} 3,{ }_{-} 5,{ }_{-} 0\right) \\
& +0.5 x_{-}\left(2,{ }_{-} 3,{ }_{-} 5,-1\right)+0.5 x_{-}\left(2,{ }_{-} 3,{ }_{-} 5,{ }_{-} 2\right)+0.2 x_{-}\left(2,{ }_{-} 3,{ }_{-} 6,{ }_{-} 0\right) \\
& +0.5 x_{-}\left(2,{ }_{-} 3,{ }_{-} 6, \_1\right)+0.5 x_{-}\left(2,{ }_{-} 3,{ }_{-} 6, x_{-} 2\right)-2 x_{-}\left(2,{ }_{-} 4,{ }_{-} 0,{ }_{-} 0\right) \\
& -5 x_{-}\left(2, \_4, \_0, \_1\right)-5 x_{-}\left(2,{ }_{-} 4, \_0, \_2\right)-2 x_{-}\left(2,{ }_{-} 4, \_1,-0\right)-5 x_{-}\left(2,{ }_{-} 4,{ }_{-} 1,{ }_{-} 1\right) \\
& -5 x_{-}\left(2,,_{-} 4,{ }_{-} 1,2\right)+0.2 x_{-}\left(2,{ }_{-} 4,{ }_{-} 2,{ }_{-} 0\right)+0.5 x_{-}\left(2,{ }_{-} 4,{ }_{-} 2, \_1\right)
\end{aligned}
$$

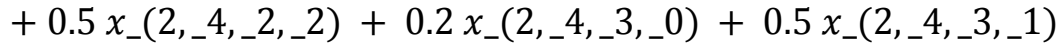

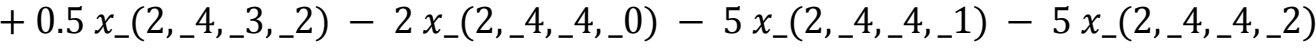

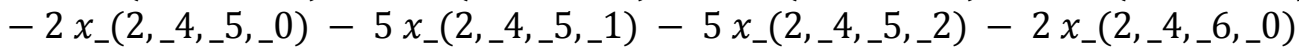

$$
\begin{aligned}
& -5 x_{-}\left(2,{ }_{-} 4, \_6, \_1\right)-5 x_{-}\left(2,{ }_{-} 4, \_6, \_2\right)+0.2 x_{-}\left(2,{ }_{2} 5,{ }_{-} 0,,_{-} 0\right)
\end{aligned}
$$

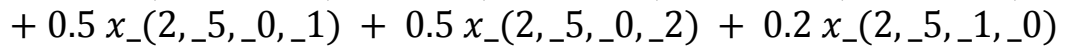

$$
\begin{aligned}
& +0.5 x_{-}\left(2,{ }_{-} 5, \_1, \_1\right)+0.5 x_{-}\left(2,{ }_{-} 5,{ }_{-} 1,{ }_{-} 2\right)-2 x_{-}\left(2,{ }_{-} 5,{ }_{-} 2,{ }_{-} 0\right)
\end{aligned}
$$

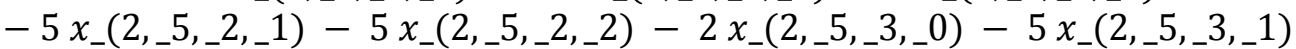

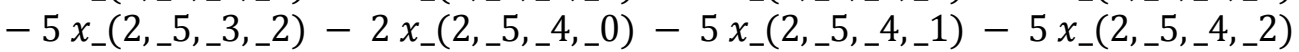

$$
\begin{aligned}
& -2 x_{-}\left(2,{ }_{-} 5, \_5, \_0\right)-5 x_{-}\left(2,{ }_{-} 5, \_5,-1\right)-5 x_{-}\left(2,{ }_{-} 5, \_5, \_2\right)-2 x_{-}\left(2, \_5, \_6, \_0\right) \\
& -5 x_{-}\left(2, \_5, \_6, \_1\right)-5 x_{-}\left(2, \_5, \_6, \_2\right)
\end{aligned}
$$

\section{RESTRICCIONES}

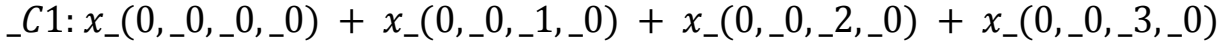

$$
\begin{aligned}
& +x_{-}\left(0,0_{-} 0,{ }_{-} 4, \_0\right)+x_{-}\left(0,{ }_{-} 0,{ }_{-} 5,{ }_{-} 0\right)+x_{-}\left(0,{ }_{-} 0,{ }_{-} 6,{ }_{-} 0\right)<=1
\end{aligned}
$$

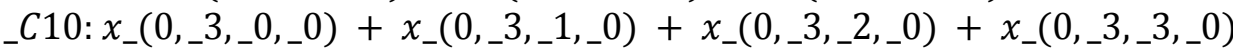

$$
\begin{aligned}
& +x_{-}\left(0,{ }_{-} 3,{ }_{-} 4,{ }_{-} 0\right)+x_{-}\left(0,{ }_{-} 3,{ }_{-} 5,{ }_{-} 0\right)+x_{-}\left(0,{ }_{-} 3,{ }_{-} 6,{ }_{-} 0\right)<=1
\end{aligned}
$$

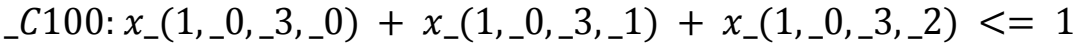

$$
\begin{aligned}
& \text { _C101: } x_{-}\left(1,1_{-} 0,{ }_{-} 4,{ }_{-} 0\right)+x_{-}\left(1,1_{-} 0,,_{-} 4, \_1\right)+x_{-}\left(1,1_{-} 0,{ }_{-} 4, x_{-} 2\right)<=1 \\
& \text { _C102: } x_{-}\left(1,1_{-} 0, \_5,{ }_{-} 0\right)+x_{-}\left(1,1_{-} 0,{ }_{-} 5, \_1\right)+x_{-}\left(1,1_{-} 0,{ }_{-} 5, \_2\right)<=1 \\
& \text { _C103: } x_{-}\left(1,1_{-} 0,{ }_{-} 6,{ }_{-} 0\right)+x_{-}\left(1,1_{-} 0,{ }_{-} 6,{ }_{-} 1\right)+x_{-}\left(1,1_{-} 0,{ }_{-} 6, x_{-} 2\right)<=1
\end{aligned}
$$

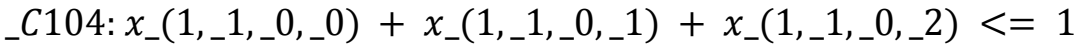

$$
\begin{aligned}
& \text { _C105: } x_{-}\left(1,1_{-} 1,1_{-} 1,0\right)+x_{-}\left(1,1_{-} 1,{ }_{-} 1,{ }_{-} 1\right)+x_{-}\left(1,{ }_{-} 1,1_{-} 1,{ }_{-} 2\right)<=1 \\
& \text { _C106: } x_{-}\left(1,1_{-} 1,22,0\right)+x_{-}\left(1,1_{-} 1, \_2, \_1\right)+x_{-}\left(1,{ }_{-} 1,{ }_{-} 2, \_2\right)<=1 \\
& \text { _C107: } x_{-}\left(1,1_{-} 1,33,-0\right)+x_{-}\left(1,1_{-} 1,{ }_{-} 3, \_1\right)+x_{-}\left(1,1_{-} 1,{ }_{-} 3, \_2\right)<=1 \\
& \text { _C108: } x_{-}\left(1,1_{-} 1,{ }_{-} 4,{ }_{-} 0\right)+x_{-}\left(1,1_{-} 1,{ }_{-} 4,{ }_{-} 1\right)+x_{-}\left(1,{ }_{-} 1,{ }_{-} 4,{ }_{-} 2\right)<=1 \\
& \text { _C109: } x_{-}\left(1,1_{-} 1,{ }_{-},{ }_{-} 0\right)+x_{-}\left(1,{ }_{-} 1, \_5, \_1\right)+x_{-}\left(1,{ }_{-} 1,{ }_{-} 5, \_2\right)<=1
\end{aligned}
$$




$$
\begin{aligned}
& +x_{-}\left(1, \_3, \_1,-2\right)+x_{-}\left(1, \_3, \_2, \_2\right)+x_{-}\left(1,{ }_{-} 3, \_3, \_2\right)+x_{-}\left(1,{ }_{-} 3,{ }_{-} 4,{ }_{-} 2\right) \\
& +x_{-}\left(1,1_{-} 3,{ }_{-} 5,{ }_{-} 2\right)+x_{-}\left(1,{ }_{-} 3, \_6,{ }_{-} 2\right)+x_{-}\left(1,{ }_{-} 4,{ }_{-} 0,{ }_{-} 2\right)+x_{-}\left(1,{ }_{-} 4,{ }_{-} 1,{ }_{-} 2\right) \\
& +x_{-}\left(1,{ }_{-} 4,{ }_{-} 2,{ }_{-} 2\right)+x_{-}\left(1,{ }_{-} 4,{ }_{-} 3,{ }_{-} 2\right)+x_{-}\left(1,{ }_{-} 4,{ }_{-} 4,{ }_{-} 2\right)+x_{-}\left(1,{ }_{-} 4,{ }_{-} 5,{ }_{-} 2\right) \\
& +x_{-}\left(1,{ }_{-} 4,{ }_{-} 6,{ }_{-} 2\right)+x_{-}\left(1,{ }_{-} 5,{ }_{-} 0,{ }_{-} 2\right)+x_{-}\left(1,{ }_{-} 5,{ }_{-} 1,{ }_{-} 2\right)+x_{-}\left(1,{ }_{-} 5, \_2,{ }_{-} 2\right) \\
& +x_{-}\left(1,1_{-} 5,{ }_{-} 3,{ }_{-} 2\right)+x_{-}\left(1,{ }_{-} 5,{ }_{-} 4,{ }_{-} 2\right)+x_{-}\left(1,{ }_{-} 5,{ }_{-} 5,{ }_{-} 2\right)+x_{-}\left(1,{ }_{-} 5,{ }_{-} 6,{ }_{-} 2\right) \\
& +x_{-}\left(2,{ }_{-} 0,{ }_{-} 0, \_2\right)+x_{-}\left(2,{ }_{-} 0,{ }_{-} 1,{ }_{-} 2\right)+x_{-}\left(2,{ }_{-} 0,{ }_{-} 2,{ }_{-} 2\right)+x_{-}\left(2,{ }_{-} 0,{ }_{-} 3, \_2\right) \\
& +x_{-}\left(2,{ }_{-} 0,{ }_{-} 4,{ }_{-} 2\right)+x_{-}\left(2,{ }_{-} 0,{ }_{-} 5,{ }_{-} 2\right)+x_{-}\left(2,{ }_{-} 0,{ }_{-} 6,{ }_{-} 2\right)+x_{-}\left(2,{ }_{-} 1,{ }_{-} 0,{ }_{-} 2\right) \\
& +x_{-}\left(2, \_1,1_{-} 1,2\right)+x_{-}\left(2, \_1,{ }_{-} 2,-2\right)+x_{-}\left(2,{ }_{-} 1,{ }_{-} 3, x_{-} 2\right)+x_{-}\left(2,{ }_{-} 1,{ }_{-} 4,{ }_{-} 2\right) \\
& +x_{-}\left(2,,_{-} 1, \_5, \_2\right)+x_{-}\left(2,{ }_{-} 1,{ }_{-} 6,{ }_{-} 2\right)+x_{-}\left(2,{ }_{-} 2,{ }_{-} 0, \_2\right)+x_{-}\left(2,{ }_{-} 2,{ }_{-} 1, \_2\right) \\
& +x_{-}\left(2, \_2, \_2,-2\right)+x_{-}\left(2, \_2, \_3, \_2\right)+x_{-}\left(2, \_2,{ }_{-} 4, \_2\right)+x_{-}\left(2,{ }_{-} 2, \_5, \_2\right) \\
& +x_{-}\left(2, \_2, \_6, \_2\right)+x_{-}\left(2, \_3, \_0, \_2\right)+x_{-}\left(2, \_3, \_1, \_2\right)+x_{-}\left(2, \_3, \_2, \_2\right) \\
& +x_{-}\left(2,{ }_{-} 3, \_3, \_2\right)+x_{-}\left(2,{ }_{-} 3,{ }_{-} 4, \_2\right)+x_{-}\left(2,{ }_{-} 3, \_5, \_2\right)+x_{-}\left(2,{ }_{-} 3, \_6, \_2\right) \\
& +x_{-}\left(2,,_{-} 4,{ }_{-} 0,{ }_{-} 2\right)+x_{-}\left(2,,_{-} 4,{ }_{-} 1,{ }_{-} 2\right)+x_{-}\left(2,{ }_{-} 4,{ }_{-} 2,{ }_{-} 2\right)+x_{-}\left(2,{ }_{-} 4,{ }_{-} 3,{ }_{-} 2\right) \\
& +x_{-}\left(2,{ }_{-} 4,{ }_{-} 4,{ }_{-} 2\right)+x_{-}\left(2,{ }_{-} 4,{ }_{-} 5,{ }_{-} 2\right)+x_{-}\left(2,{ }_{-} 4,{ }_{-} 6,{ }_{-} 2\right)+x_{-}\left(2,{ }_{-} 5,{ }_{-} 0,{ }_{-} 2\right) \\
& +x_{-}\left(2, \_5, \_1, \_2\right)+x_{-}\left(2, \_5, \_2,-2\right)+x_{-}\left(2,{ }_{-} 5, \_3, \_2\right)+x_{-}\left(2, \_5, \_4, \_2\right) \\
& +x_{-}\left(2,{ }_{-} 5, \_5, \_2\right)+x_{-}\left(2, \_5, \_6, \_2\right)=6
\end{aligned}
$$

